\title{
220-MHz NMR Spectra of Methyl Methacrylate-1,3-Diolefin Alternating and Random Copolymers
}

\author{
Toshimitsu Suzuki, Katsuo Mitani, and Yoshinobu Takegami \\ Department of Hydrocarbon Chemistry, Faculty of Engineering, \\ Kyoto University, Kyoto 606, Japan. \\ Junji Furukawa \\ Department of Synthetic Chemistry, Faculty of Engineering, \\ Kyoto University, Kyoto 606, Japan.
}

Eiichi Kobayashi and Yoshihiro AraI

Institute for Chemical Research, Kyoto University, Uji 611, Japan.

(Received February 4, 1974)

\begin{abstract}
A 220-MHz NMR investigation was carried out on the random and alternating copolymers of methyl methacrylate (MMA) with butadiene (BD) or isoprene (IP) and their partially deuterated analogues. An absorption by the $\alpha$-methyl protons of MMA units in the MMA-BD random copolymer splits into several overlapping peaks. The triad sequence distributions centered at the MMA unit were roughly estimated and compared with those calculated from the first-order Markov chain model, providing that the monomer reactivity ratios were $r_{\mathrm{MMA}}=0.18$ and $r_{\mathrm{BD}}=0.62$. The observed and calculated sequence distribution for the random copolymer are in fair agreement. From the resonance mode of the $\alpha$-methyl proton of MMA-Bd- $d_{4}$ alternating copolymer, an isotactic configuration with flanking BD units was suggested. The alternating copolymer contains more than $95 \%$ of the alternating MMA-BD triad.

Almost the same spectrum was observed for the MMA-IP alternating copolymer, and the chain structure was established to be composed of a linkage between the $\alpha$ position of MMA and the 1-position of IP. To determine the monomer sequence distribution of MMA-IP random copolymers, the lanthanide NMR shift reagent was used to separate the overlapped peaks. The monomer sequence distribution of the random copolymer obeys the first-order Markov chain model, providing that the monomer reactivity ratios are $r_{\mathrm{MMA}}=0.26$ and $r_{\mathrm{IP}}=0.625$. The alternating copolymer contains more than $97 \%$ of the MMA-IP alternating triad.

KEY WORDS 220-MHz NMR / Copolymer / Alternating Copolymer / Butadiene / Isoprene / Deuterated Diene / Methyl Methacrylate / Sequence Distribution / Markov Chain / Structure of Copolymer /
\end{abstract}

In the previous papers some of the present authors have reported both an alternating copolymerization of acrylic monomers and conjugated diene with a catalyst of alkylaluminum halide-transition metal compound and the characterization of these alternating copolymers by means of infrared and ${ }^{1} \mathrm{H}-\mathrm{NMR}$ analyses. ${ }^{1,2}$ According to the $220-\mathrm{MHz}$ NMR spectra of the alternating copolymer of acrylonitrile and butadiene, ${ }^{3}$ the amount of alternating triad sequence was more than 98\%. The triad sequence was estimated from the methyne proton resonance of the acrylonitrile units, whereas the pentad sequence was evaluated from the olefin proton resonance of the butadiene units. If the amount of triad and pentad sequences in the alternating copolymer of acrylonitrile and butadiene can be quantitatively determined, the data will offer information about the polymerization mechanism. However, the nonalternating pentad se- 
quence was negligibly small and could not be determined quantitatively. ${ }^{3}$ On the other hand, the vicinal coupling constant of the alternating copolymer of acrylonitrile and deuterated butadiene showed a somewhat twisted trans molecular structure conformation ${ }^{3}$ such as was also suggested by the infrared dichroic measurement and X-ray diffraction diagram of the stretched copolymer film. ${ }^{4}$

There are several $100-\mathrm{MHz}$ NMR studies of alternating copolymers of methyl methacrylatebutadiene, ${ }^{2}$ methyl methacrylate-isoprene, ${ }^{5}$ acrylonitrile-butadiene ${ }^{6}$ and acrylonitrile-isoprene, ${ }^{6}$ and $220-\mathrm{MHz}$ NMR studies of methyl methacrylate-isoprene ${ }^{7}$ and acrylonitrile-butadiene. ${ }^{3}$ However, these did not make full assignment of the spectra. The $300-\mathrm{MHz}$ NMR spectrum of the alternating copolymer of acrylonitrile and butadiene was reported by Harwood, et $a .^{8} \quad$ A considerable reduction in overlapping of resonance spectra was observed. They assigned the olefin protons of the butadiene unit flanked by acrylonitrile as $d d$ or $l l$ configurations with $d l$ or $l d$ ones of nearly equal intensity and concluded that the meso and racemic arrangements of the acrylonitrile units were equally probable. ${ }^{8}$

In the present paper the authors studied a $220-\mathrm{MHz}$ NMR spectrum of methyl methacrylate and butadiene or isoprene to elucidate the sequence distribution, the 1,4 or 4,1 arrangement of isoprene and the stereospecific configuration of the monomer units. In some cases the assignment of spectra was carried out either by the use of the deuterated monomer or by the use of the lanthanide NMR shift reagent. ${ }^{9-11}$

\section{EXPERIMENTAL}

\section{Materials}

Butadiene (BD) and isoprene (IP) were purified by the usual methods. Methyl methacrylate (MMA) was washed with dilute alkali solution, dried over calcium hydride and distilled just before use. Ethylaluminum dichloride $\left(\mathrm{EtAlCl} \mathrm{Al}_{2}\right)$ was distilled under reduced pressure and used as a $n$-hexane solution. Vanadyl chloride $\left(\mathrm{VOCl}_{3}\right)$ was used as a $n$-hexane solution. 1,1 , 4,4- $d_{4}$-Butadiene (BD- $d_{4}$ ) and 1,1,4,4- $d_{4}$-isoprene (IP- $\left.d_{4}\right)$ were prepared from the $\mathrm{H}-\mathrm{D}$ exchange reaction of the cyclic sulfones of the respective 1,3-diene and deuterium oxide followed by thermal decomposition. ${ }^{12}$

\section{Polymerization Procedures}

Alternating copolymers were prepared as follows; MMA, diene, EtAlCl 2 and $\mathrm{VOCl}_{3}$ were introduced in that order at $-78^{\circ} \mathrm{C}$ into an ampule flushed with purified nitrogen, and the

Table I. Synthesis of copolymers

\begin{tabular}{|c|c|c|c|c|c|c|}
\hline \multirow{2}{*}{ No. } & \multirow{2}{*}{$\begin{array}{l}\text { Feed ratio, } \\
\text { MMA/Diene }\end{array}$} & \multicolumn{2}{|c|}{ Polymerization } & \multirow{2}{*}{ Cat.a } & \multirow{2}{*}{$\begin{array}{c}\text { Yield, } \\
\%\end{array}$} & \multirow{2}{*}{$\begin{array}{l}\text { Polymer composition, } \\
\text { MMA in copolymer, } \\
\text { mole fraction }\end{array}$} \\
\hline & & Temp, ${ }^{\circ} \mathrm{C}$ & Time, hr & & & \\
\hline BD-1 & 9.0 & 50 & 4.0 & B & 2.2 & $0.73_{0}$ \\
\hline BD-2 & 1.0 & 50 & 11.0 & B & 3.6 & $0.44_{1}$ \\
\hline BD-3 & 0.17 & 50 & 10.0 & B & 5.8 & $0.19_{6}$ \\
\hline BD-4 & 1.0 & 0 & 20.0 & V & 81.0 & 0.50 \\
\hline BD-5c & 1.0 & 0 & 4.0 & V & 63.0 & 0.50 \\
\hline IP-1 ${ }^{c}$ & 0.224 & 50 & 12.0 & B & 9.7 & $0.21_{4}$ \\
\hline IP-2c & 0.663 & 50 & 11.0 & B & 13.9 & $0.40_{5}$ \\
\hline $\mathrm{IP}^{-3^{\mathrm{c}}}$ & 1.40 & 50 & 6.0 & B & 9.6 & $0.48_{6}$ \\
\hline IP-4 $4^{\mathrm{C}}$ & 2.34 & 50 & 5.0 & B & 10.2 & $0.55_{2}$ \\
\hline IP-5c & 7.0 & 50 & 4.5 & B & 12.6 & $0.70_{9}$ \\
\hline IP-6 & 1.0 & 0 & 20.0 & Z & 15.0 & 0.50 \\
\hline IP-7c & 1.0 & 0 & 20.0 & Z & 20.0 & 0.50 \\
\hline IP-8 & 1.0 & 0 & 20.0 & V & 6.7 & 0.50 \\
\hline
\end{tabular}

a $\mathrm{B}, \mathrm{BPO}$ as catalyst; $\mathrm{V}, \mathrm{AlEtCl}_{2}-\mathrm{VOCl}_{3}$ as catalyst; $\mathrm{Z}, \mathrm{ZnCl}_{2}-\mathrm{MMA}$ complex was used.

$\mathrm{b}$ Determined from elemental analysis.

c BD- $d_{4}$ or IP- $d_{4}$ monomer was used. 
ampule was sealed off. After a certain reaction time at $0^{\circ} \mathrm{C}$ the copolymer was precipitated by pouring it into a large amount of methanol. An alternating copolymer of MMA-IP was prepared with zinc chloride according to the method described by Oikawa, et al. ${ }^{5}$ Random copolymers were prepared with benzoyl peroxide (BPO) as an initiator at $50^{\circ} \mathrm{C}$ in the bulk. The polymerization conditions and the results are summarized in Table I.

\section{NMR Spectra}

NMR spectra were obtained using a Varian HR 220 spectrometer. Approximately 5-7\% (wt/vol) deuterochloroform solutions of the copolymers were employed with tetramethylsilane as an internal reference. The temperature for the measurements was set at $60^{\circ} \mathrm{C}$. Peak areas were determined by cutting out and weighing Xerox copies of the spectrum sheets. Commercial Pr-1,1,1,2,2,3,3-heptafluoro-7,7-dimethyl4,6-octanedione $\left(\operatorname{Pr}(\mathrm{fod})_{3}\right)$ was used as a shift reagent.

\section{RESULTS AND DISCUSSION}

\section{Methyl Methacrylate-Butadiene Alternating and Random Copolymers}

In Figure 1 (BD-5) the $220-\mathrm{MHz}$ NMR spectrum of MMA-BD- $d_{4}$ alternating copolymers is presented. A sharp singlet at $1.095 \delta$ is assigned to the $\alpha$-methyl proton of the MMA units in the BD-MMA-BD triad sequence, and weak absorptions at $0.986 \delta, 1.023 \delta$ and a shoulder at $1.109 \delta$ may be assigned to irregular sequences contained in the alternating copolymer. The relative peak areas at $0.986,1.023,1.095$ (main) and $1.109 \delta$ are $0.02,0.03,1.00$, and 0.12 , respectively. These will be discussed in the following section. The $\mathrm{AB}$ quartet peaks centered at $1.421 \delta$ and $1.628 \delta$ are attributed to the methylene protons of the MMA units in the alternating sequences $\left(J_{A B}=-13.6 \mathrm{~Hz}\right.$ and $\delta_{A}-$ $\delta_{\mathrm{B}}=45.9 \mathrm{~Hz}$ ). A sharp singlet at $3.638 \delta$ is the methoxy proton of the MMA units, and the $\mathrm{AB}$ quartet peaks centered at $5.291 \delta$ and 5.377 $\delta$ can be assigned to the trans olefin protons of the BD- $d_{4}$ units in the alternating sequences $\left(J_{\mathrm{AB}}=15.0 \mathrm{~Hz}\right.$ and $\left.\delta_{\mathrm{A}}-\delta_{\mathrm{B}}=19.0 \mathrm{~Hz}\right)$. Harwood, et al., ${ }^{8}$ using a $300-\mathrm{MHz}$ spectrometer, demon- strated that two sets of $\mathrm{AB}$ quartet peaks of nearly equal intensities are involved in the olefin protons of the acrylonitrile (AN)-BD alternating copolymer. They attributed one $A B$ pattern to the $d d$ or $l l$ configuration of $\mathrm{AN}$ units flanked by BD- $d_{4}$ units and the other to the other to the $d l$ or $l d$ configuration. This means that the AN-BD alternating copolymer has a random configuration of AN units. However, in this MMA-BD- $d_{4}$ alternating copolymer the olefin protons show one major $\mathrm{AB}$ pattern and at the low field side of each peak small shoulder peaks are observed. According to the infrared analyses, ${ }^{13}$ the copolymer shows characteristic absorptions corresponds to a probable isotactic configuration of the MMA units. Therefore, these features in the NMR spectrum of the MMABD- $d_{4}$ alternating copolymer indicate that the major AB pattern can be attributed to an alternating $l l$ and/or $d d$ sequence of MMA units.

If one assumes the minor quartet peaks due to olefin protons are an occasional irregular sequence such as $\mathrm{MMA}-\mathrm{BD}-\mathrm{BD}$ or $\mathrm{BD}-\mathrm{BD}-$ $\mathrm{BD}$, then these peaks should appear at a lower field judging from the copolymer rich in $\mathrm{BD}$ units (see Figure 2). This means that the olefin proton resonance gives no direct information about the present situation with regard to the configuration and irregular sequence. On the other hand, for the resonance of the $\alpha$-methyl protons of the MMA units, a relatively large amount of shoulder peak was observed at 1.109 $\delta$. This peak should be attributed to the MMABD-MMA-BD-BD and/or BD-BD-MMA $-\mathrm{BD}-\mathrm{BD}$ pentad or the $l d$ and/or $d l$ configuration of the MMA units in the alternating sequences. If one takes the former assignment, the copolymer probably contains a considerable amount of MMA-MMA-BD or MMA-MMA -MMA triad, because the composition of the copolymer is essentially $1: 1$. These triad peaks can be found at $0.986 \delta$ and $1.023 \delta$, but the intensities of these peaks are smaller than those expected from the peak at $1.109 \delta$. Thus the shoulder peak at $1.109 \delta$ can be attributed to the $d l$ and/or $l d$ configuration of MMA units but not the irregular pentad as above mentioned.

The methylene protons of isotactic poly(methyl methacrylate) exhibit a nonequivalent $\mathrm{AB}$ 


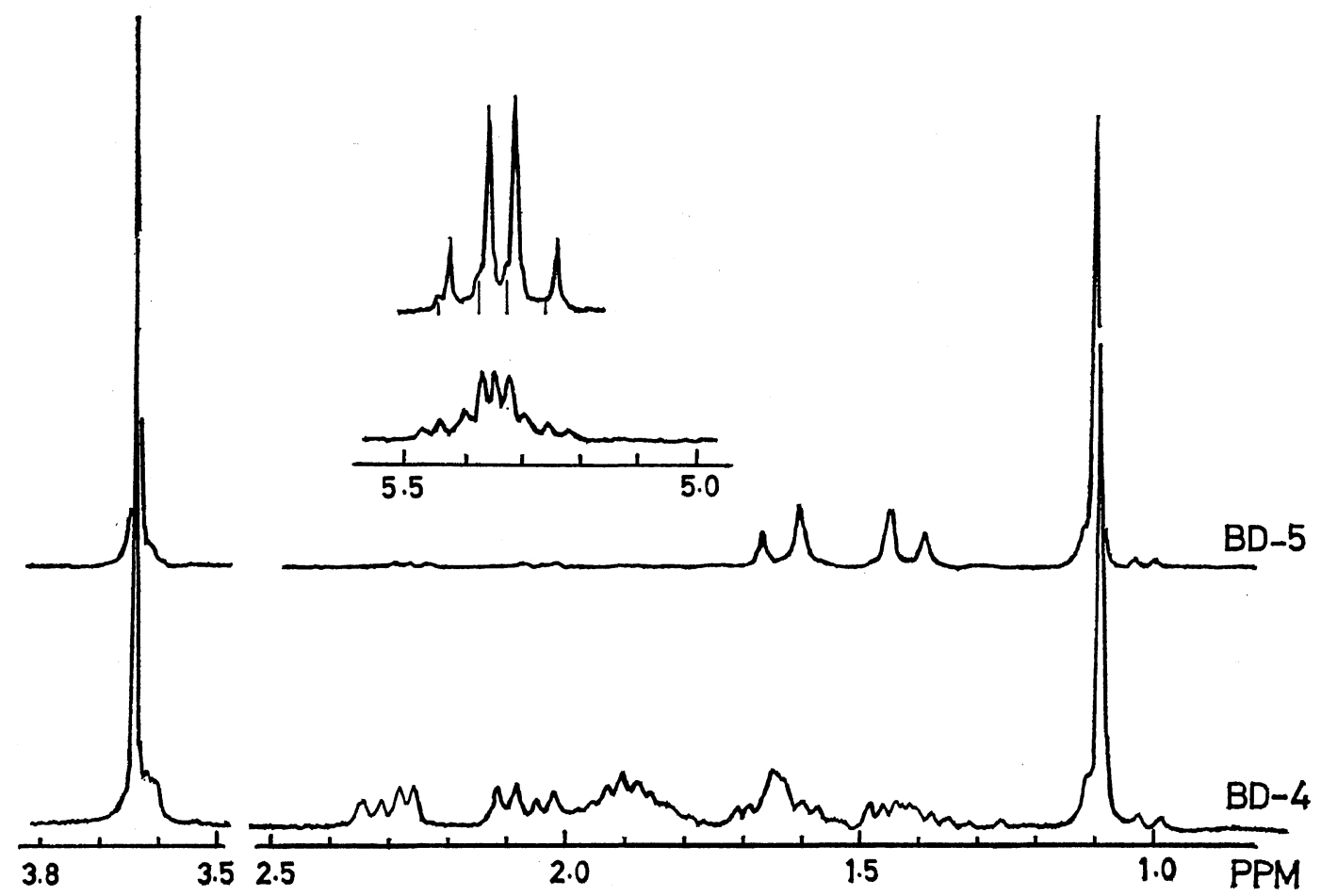

Figure 1. NMR spectra of MMA-BD (BD-4) and MMA-BD- $d_{4}$ (BD-5) alternating copolymer.

quartet. $^{14}$ The difference in the chemical shift between threo and erythro protons is reported to be $0.7 \mathrm{ppm}$. On the other hand, the difference in the chemical shift of the MMABD- $d_{4}$ alternating copolymer is only $0.2 \mathrm{ppm}$. In Figure 1 (BD-4) the NMR spectrum of MMA-BD alternating copolymer is presented. Comparing this with the spectrum of MMABD- $d_{4}$ alternating copolymer, the absorptions at $1.91 \delta, 2.05 \delta$ and $2.3 \delta$ are assigned to the methylene protons of the BD units. The integrated intensities of these absorptions are $2: 1: 1$, respectively, and they are assigned as follows:

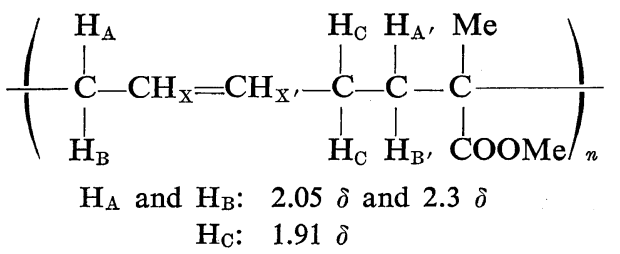

$H_{A}$ and $H_{B}$ are coupled to $H_{X}$, are nonequivalent with respect to the adjacent asymmetric $\alpha$ carbon of the MMA units and accordingly show an $A B X$ spin system pattern. On the other hand, the $\mathrm{H}_{\mathrm{C}}$ proton shows a very complicated pattern. The methylene protons of the MMA units are represented as an $\mathrm{ABC}_{2}$ pattern and an absorption of unknown irregular sequence appeared at $1.65 \delta$.

MMA-BD copolymers of various composition were prepared by a free radical mechanism. The NMR spectra of some of them are shown in Figure 2. Resonance spectra of the $\alpha$-methyl protons of MMA units are separated into several peaks with deep overlapping. This feature is quite similar to that reported in the copolymer of MMA-IP. ${ }^{7}$ With increasing amounts of MMA content in the copolymer, the relative intensity of the higher field peaks increases. This means that the $\alpha$-methyl proton resonance of the central MMA unit shifts upfield when the central MMA unit is surrounded by MMA units. In poly(methyl methacrylate) $\alpha$-methyl proton splits into three major peaks corresponding to the $\mathrm{mm}(1.22 \delta), \mathrm{mr}(1.06 \delta)$ and $\operatorname{rr}(0.89$ $\delta)$ triads. ${ }^{14}$ Accordingly, in this copolymer the absorption of $\alpha$-methyl protons in the MMAMMA-MMA triads are split into three major 


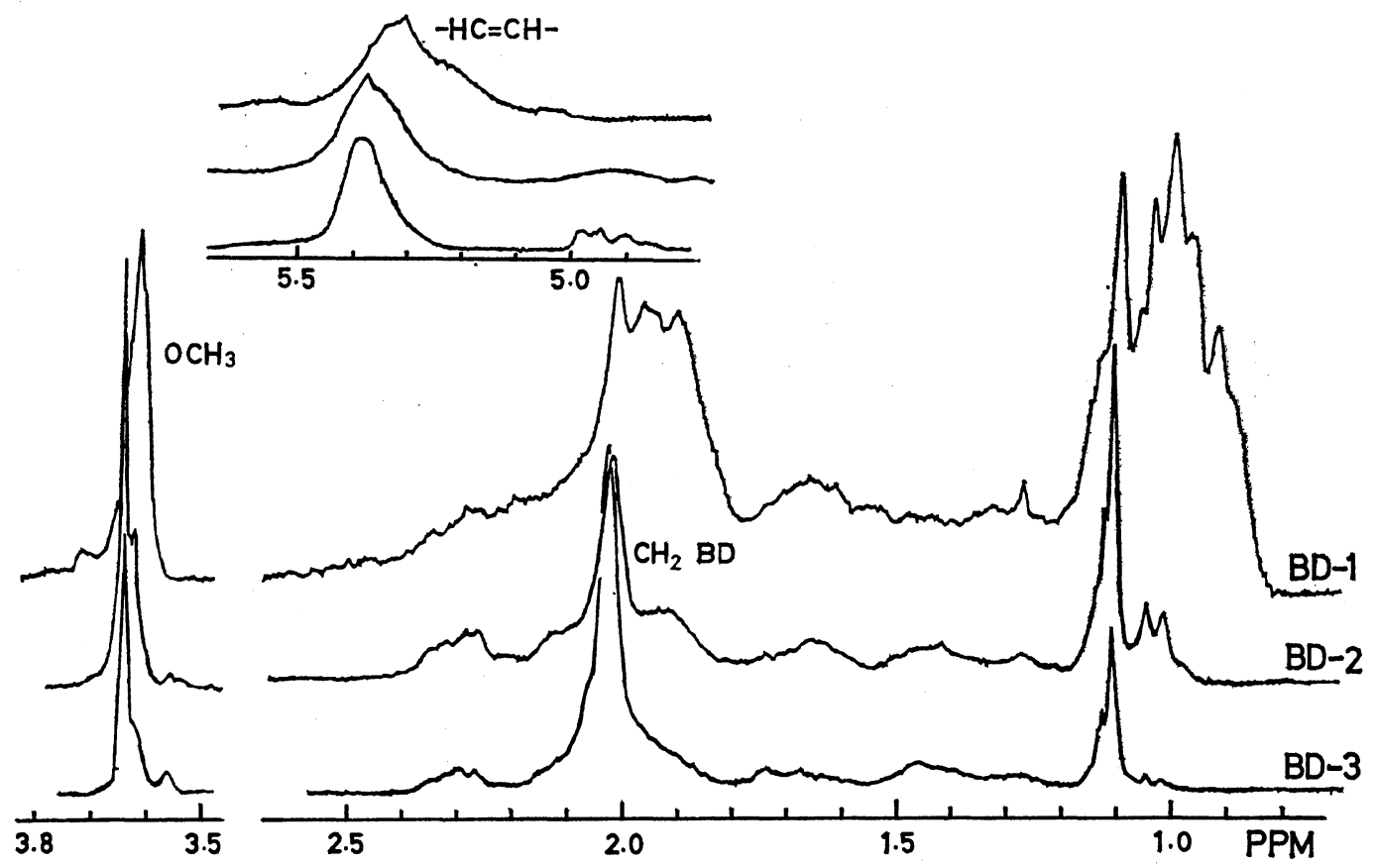

Figure 2. NMR spectra of MMA-BD random copolymers.

Table II. Monomer-sequence distribution of MMA-BD random copolymers in mole fraction

\begin{tabular}{|c|c|c|c|c|c|c|}
\hline & \multicolumn{2}{|c|}{ BD-1 } & \multicolumn{2}{|c|}{ BD-2 } & \multicolumn{2}{|c|}{ BD-3 } \\
\hline & Obsd & Calcd $^{\mathrm{a}}$ & Obsd & Calcd & Obsd & Calcd \\
\hline MMA in copolm. & 0.730 & 0.710 & 0.441 & 0.421 & 0.196 & 0.186 \\
\hline \multicolumn{7}{|l|}{ Sequence } \\
\hline MMA-MMA-MMA & 0.447 & 0.383 & 0.050 & 0.026 & 0.0 & 0.0 \\
\hline MMA-MMA-BD & 0.399 & 0.472 & 0.253 & 0.284 & 0.105 & 0.060 \\
\hline BD-MMA-BD & 0.143 & 0.146 & 0.700 & 0.650 & 0.895 & 0.939 \\
\hline
\end{tabular}

a Calculated from a first-order Markov chain model taking the monomer reactivity ratios to be $r_{\mathrm{MMA}}=$ 0.18 and $r_{\mathrm{BD}}=0.62$.

peaks by the triad stereosequences. In addition, the MMA-MMA-BD sequence is also affected by the stereoarrangement of the MMA-MMA units. Therefore, it is very difficult to determine the monomer sequence distribution of the copolymer with a large fraction of MMA units. The monomer sequence distribution was estimated roughly from the assumption that the triad tacticity was the same as that obtained from homopolymerization of MMA at $50^{\circ} \mathrm{C}$ with $\mathrm{BPO}$ as an initiator and possessing $\mathrm{mm}=$ $0.07, \mathrm{mr}=0.33$ and $\mathrm{rr}=0.60$. Absorptions of the $\alpha$-methyl resonances were divided into three region (i) $0.94 \delta$ and up, (ii) $0.94-1.05 \delta$ and (iii) $1.05-1.33 \delta$. Upon correcting the MMAMMA-MMA triad peaks for the $\mathrm{mr}$ and $\mathrm{mm}$ triad within region (iii), the relative number of monomer sequences of MMA centered in the triad were obtained. The observed and calculated monomer sequence distributions are listed in Table II. The monomer sequence distributions calculated from the monomer reactivity ratios $r_{\mathrm{MMA}}=0.18$ and $r_{\mathrm{BD}}=0.62,{ }^{15}$ fit the observed ratios fairly well. Therefore, the copolymerization of MMA and BD by BPO obeys a first-order Markov chain mechanism. Ac- 
cording to the above spectral assignment, the amount of alternating triad sequence is more than $95 \%$.

\section{Methyl Methacrylate-Isoprene Alternating and Rand Copolymers}

For copolymers of this kind two types of structure are possible, i.e., I and II.<smiles>CCC(C)=CCC(C)C(C)CC</smiles>
I<smiles>CCC=C(C)C(C)C(C)(C)CC</smiles>

II

This difference is concerned with the mode of addition of isoprene to the $\alpha$-position of MMA; the 4-addition results in structure $I$, whereas the 1-addition, structure II. (In $\mathrm{C}_{6} \mathrm{D}_{6}$ with peaks at 2.68 and $2.54 \delta$ in a $100-\mathrm{MHz}$ spectra,
Oikawa, et al., propose $\mathbf{I}$ as the structure. ${ }^{5}$ )

Figure 3 shows the NMR spectra of MMAIP alternating copolymers. These spectra are very similar to those of MMA-BD alternating copolymers. Comparing with MMA-IP- $d_{4}$ copolymer (Figure 3 IP-7) the assignments of the peaks are carried out as follows: Part of the $\mathrm{AB}$ quartet peaks at $2.45 \delta$ and the other part at 2.0-2.1 $\delta$ are assigned to $\mathrm{H}_{\mathrm{A}}$ or $\mathrm{H}_{B}$ methylene protons of the IP units in structure Il. An absorption by the other methylene proton of the IP units appeared at $1.75-2.0 \delta$ as a complex multiplet. Methylene protons of MMA units appeared as an $\mathrm{AB}$ quartet in $\mathrm{IP}-d_{4}$ copolymer and a pattern of $\mathrm{ABC}_{2}$ spin system was observed at $1.35 \delta$ and $1.65 \delta$. Methyl protons of the IP units appeared at $1.48 \delta$ and methyl protons of the MMA units appeared at $1.08 \delta$ as sharp singlets. However, there are minor peaks due to the MMA homosequence at higher field than the $\alpha$-methyl proton of MMA units. The olefin proton of the IP unit was observed as a triplet as well as a singlet for the copolymer of IP- $d_{4}$. The methoxy

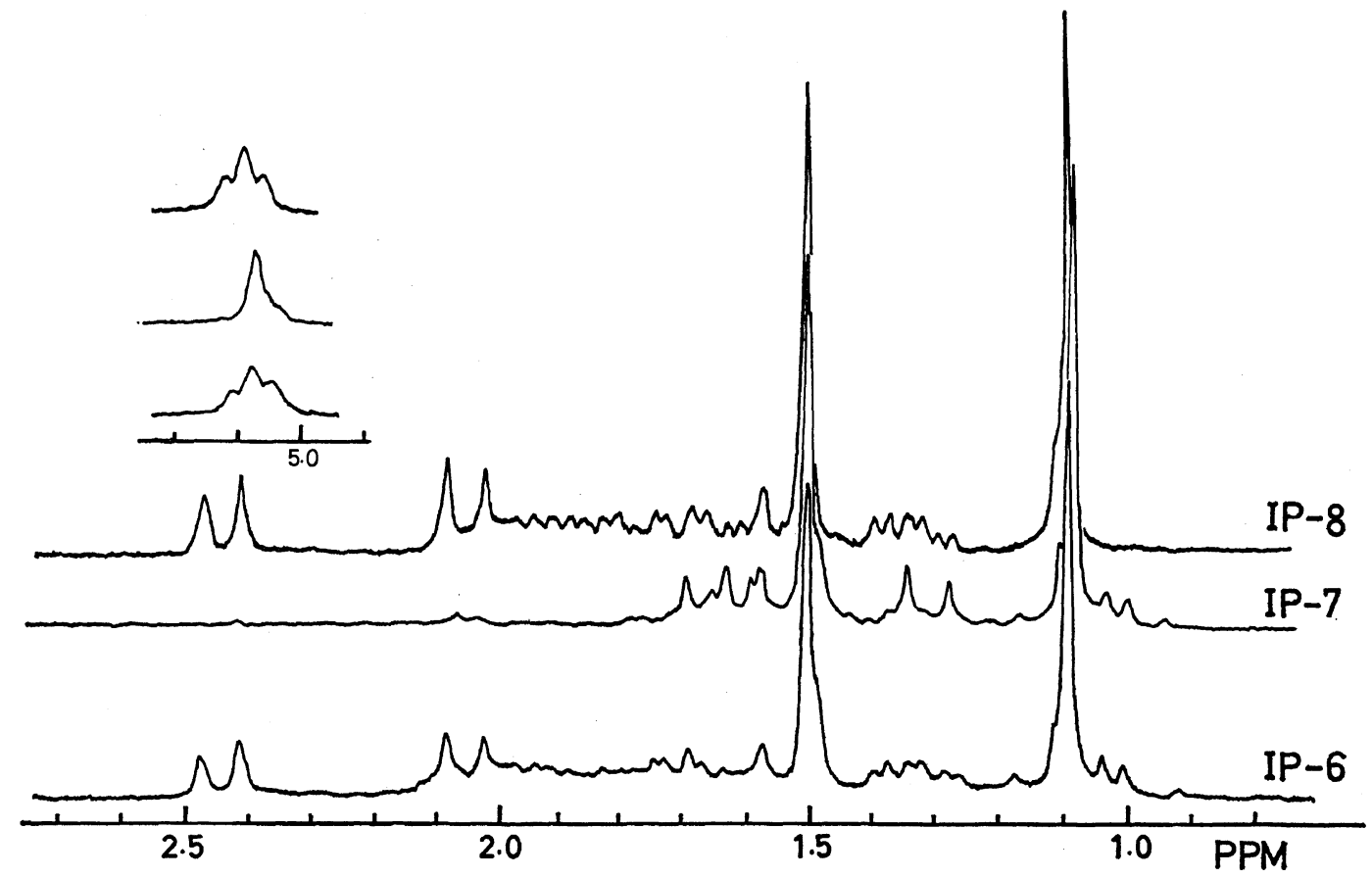

Figure 3. NMR spectra of MMA-IP alternating copolymers: IP-6, $\mathrm{ZnCl}_{2} ; \mathrm{IP}-7, \mathrm{IP}-d_{4}, \mathrm{ZnCl}_{2} ; \mathrm{IP}-$ 8, $\mathrm{EtAlCl}_{2}-\mathrm{VOCl}_{3}$. 
proton (which is not shown in the Figure) appeared at $3.65 \delta$. Previously the structure of this copolymer was presented as structure I (4-addition) by Oikawa, et al. ${ }^{5}$ They compared the NMR spectra of the alternating copolymer with the cyclic Diels-Alder adducts $\mathbf{A}$ and $\mathbf{B}$ and concluded that the cyclic adduct $\mathbf{A}$ gave a more similar spectrum to the alternating copolymer. They concluded that MMA-IP alternating copolymer might possess a chain structure such as $\mathbf{I}$.

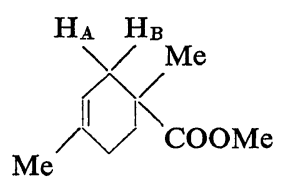

$\mathbf{A}$ $\left(2.35,2.52 \delta \text { in } \mathrm{CDCl}_{3}\right)^{5}$

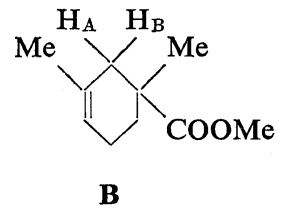

$\left(2.43,2.60 \delta \text { in } \mathrm{CDCl}_{3}\right)^{5}$

However, if one takes structure I for the alternating copolymer, $\mathrm{H}_{A}$ and $\mathrm{H}_{B}$ must couple to the $H_{X}$ proton and therefore, it is difficult to explain the reason why the isoprene methylene protons indicate the $A B$ quartet peaks of $H_{A}$ and $H_{B}$. In addition, the olefin protons of the IP units exhibit a triplet absorption, while a singlet absorption was observed when IP- $d_{4}$ was copolymerized. This clearly shows that the $\mathrm{H}_{\mathrm{X}}$ proton coupled to two equivalent protons. From these results one can easily conclude that the MMA-IP alternating copolymer has structure II.

There are many factors governing the determination of such structures. For instance, influential factors may be the steric effect of the $\alpha$-methyl group, the electron densities of the $\alpha$, $\beta$-carbons of the acrylic monomer, an interaction between the substituted groups in the 2position of the diene and a change in the nature of acrylic monomer caused by complex formation with the aluminum compound, etc. In the ${ }^{13} \mathrm{C}$ NMR spectra of an acrylic monomer complexed with a Lewis acid, ${ }^{16}$ the apparent chemical shifts of the $\alpha, \beta$-carbons of $\mathrm{AN}-\mathrm{EtAlCl}_{2}$ and $\mathrm{MMA}-\mathrm{EtAlCl}_{2}$ are 101.7, 148.9 and 134.2, 124-125 ppm from TMS, respectively. The electron density of the $\alpha$-carbon of AN is larger than that of the $\beta$-carbon, while that of the $\beta$ carbon of MMA is larger than that of the $\alpha$ - carbon. This polarization may be an influential factor for the formation of structure I or II. The bond formation between the diene and AN or MMA may occur from the reverse side with respect to the $\alpha, \beta$-carbons of acrylic monomer. This is left unsettled in this report.

Comparing the NMR spectra of the alternating copolymers prepared with $\mathrm{EtAlCl}_{2}-\mathrm{VOCl}_{3}$ and $\mathrm{ZnCl}_{2}$ as a complexing agent, the former copolymer (IP-8) exhibits a larger amount of alternating sequence than the latter (IP-6). Rough estimation of the IP-MMA-IP triad based on the $\alpha$-methyl resonance of MMA units indicated that the former copolymer and the latter one contain more than $97 \%$ and $87 \%$ of alternating triad sequence, respectively. The same results were observed in the case of the MMA-BD alternating copolymers. This may be correlated to the fact that the $\mathrm{MMA}-\mathrm{ZnCl}_{2}$ complex alone can spontaneously homopolymerize.

In Figure 4 NMR spectra of MMA-IP- $d_{4}$ random copolymers are presented. Compared with those of the alternating copolymers (see Figure 3) the resonances of the $\alpha$-methyl protons of the MMA units and the methyl protons of the IP units split into several peaks with partial overlapping. On the other hand, the olefin protons of the IP units (not shown) are not separated but a broad absorption is observed. The resonances of the methyl protons of the IP units are apparently split into three peaks. The peak in the highest field splits further into two absorptions. This may be due to pentad sequences such as MMA-MMA-IP-MMAMMA and IP-MMA-IP-MMA-MMA. Because the alternating copolymer has an absorption at lower field, as shown in Figure 4 (IP-1, i.e., the random copolymer rich in IP units) the resonances at 1.58 and $1.675 \delta$ are enhanced. These absorptions are assigned to the MMAIP_IP and IP_IP_IP (trans) triad, respectively.

To obtain the monomer sequence distribution of MMA-IP- $d_{4}$ random copolymer (IP-4), an NMR spectrum was recorded for this copolymer in a solution to which a lanthanide NMR shift reagent was added. In Figure 5 the separation of individual peak is presented for the sample with $\operatorname{Pr}(\mathrm{fod})_{3}$ shift reagent added. Resonances for the $\alpha$-methyl protons of the MMA units 


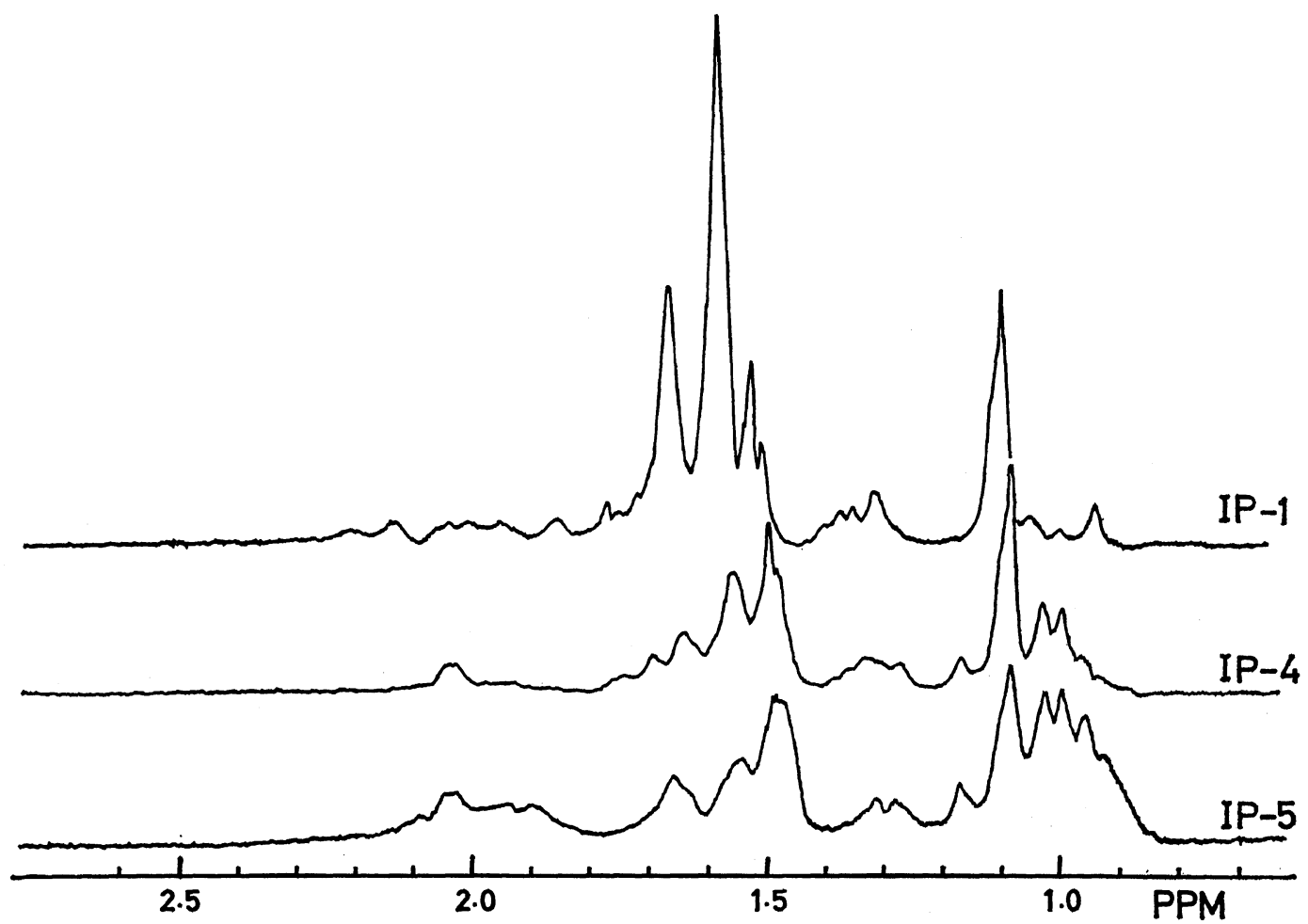

Figure 4. NMR spectra of MMA-IP- $d_{4}$ random copolymers.

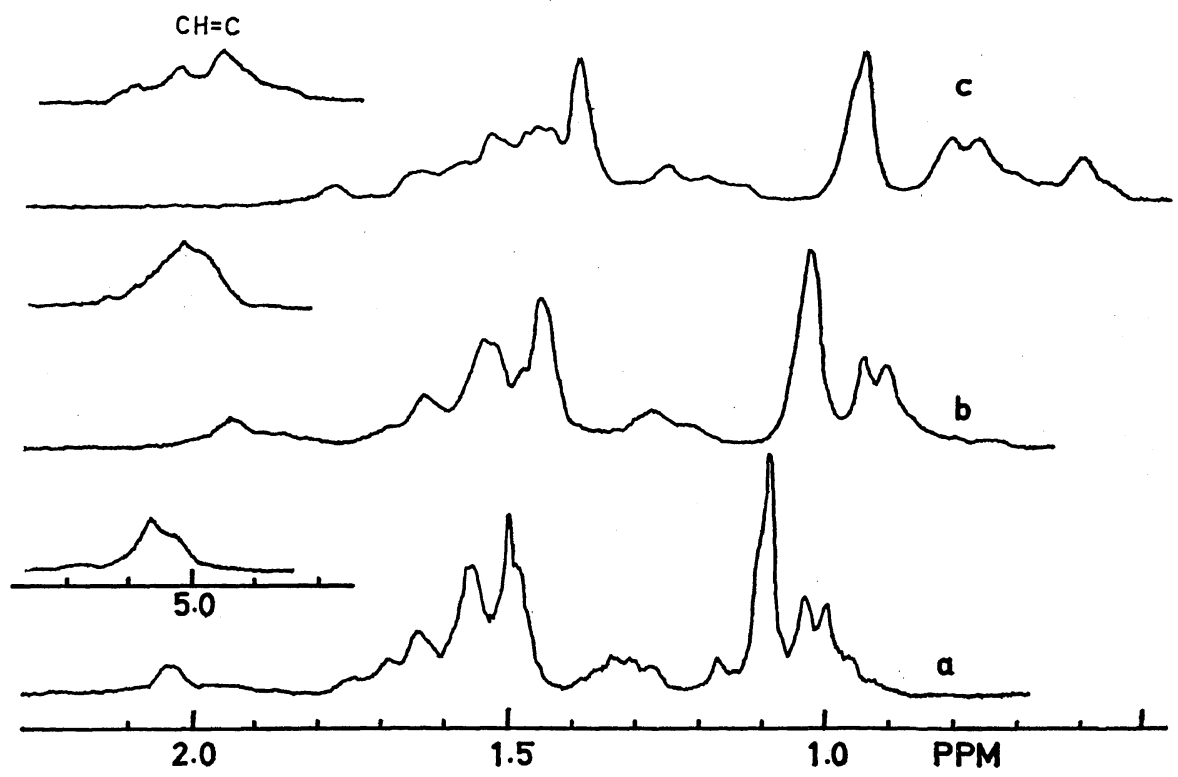

Figure 5. Lanthanide-induced shift of MMA-IP- $d_{4}$ random copolymer (IP-4): $\operatorname{Pr}(\text { fod })_{3} / \mathrm{MMA}$ unit in mole ratios: $a, 0 ; b, 0.01 ; c, 0.026$. 
T. Suzuki, K. Mitani, Y. Takegami, J. Furukawa, E. Kobayashi, and Y. Arai

Table III. Monomer-sequence distribution of MMA-IP- $d_{4}$ random copolymers in mole fraction

\begin{tabular}{|c|c|c|c|c|c|c|c|c|c|c|}
\hline & \multicolumn{2}{|c|}{ IP-1 } & \multicolumn{2}{|c|}{ IP-2 } & \multicolumn{2}{|c|}{ IP-3 } & \multicolumn{2}{|c|}{ IP-4 } & \multicolumn{2}{|c|}{ IP-5 } \\
\hline & Obsd & Calcd ${ }^{\mathrm{a}}$ & Obsd & Calcd & Obsd & Calcd & Obsd & Calcd & Obsd & Calcd \\
\hline MMA in copolm. & 0.214 & 0.218 & 0.405 & 0.376 & 0.486 & 0.486 & 0.552 & 0.559 & 0.709 & 0.722 \\
\hline \multicolumn{11}{|l|}{ Sequence } \\
\hline MMA-IP-MMA & 0.073 & 0.055 & 0.150 & 0.164 & 0.229 & 0.246 & 0.265 & 0.274 & 0.216 & 0.236 \\
\hline $\mathrm{IP}-\mathrm{IP}-\mathrm{MMA}$ & 0.288 & 0.304 & 0.256 & 0.314 & 0.193 & 0.214 & 0.128 & 0.147 & 0.059 & 0.046 \\
\hline $\mathrm{IP}-\mathrm{IP}-\mathrm{IP}$ & 0.426 & 0.424 & 0.187 & 0.147 & 0.091 & 0.048 & 0.049 & 0.020 & 0.013 & 0.002 \\
\hline IP-MMA-IP & 0.185 & 0.195 & 0.262 & 0.273 & 0.215 & 0.260 & 0.236 & 0.216 & 0.120 & 0.091 \\
\hline IP-MMA-MMA & 0.029 & 0.022 & 0.133 & 0.095 & 0.208 & 0.191 & 0.240 & 0.253 & 0.268 & 0.329 \\
\hline MMA-MMA-MMA & 0.0 & 0.0 & 0.009 & 0.008 & 0.064 & 0.035 & 0.076 & 0.080 & 0.321 & 0.301 \\
\hline
\end{tabular}

a Calculated from a first-order Markov chain model, taking the monomer reactivity ratios to be $r_{M M A}=0.26$ and $r_{I P}=0.625$. The monomer reactivity ratio was calculated from the sequence distributions of the respective run and averaged.

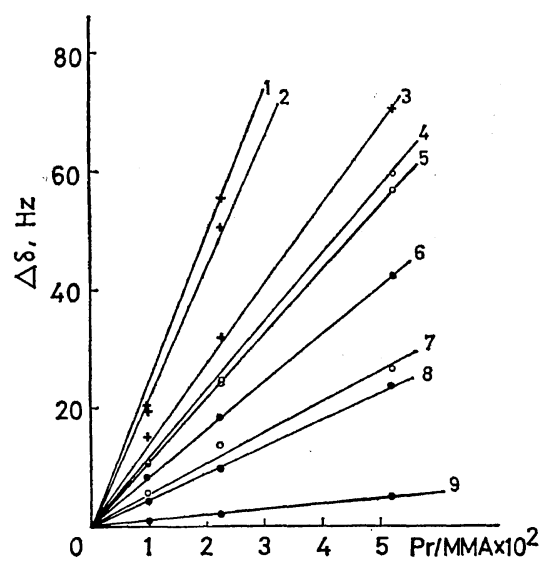

Figure 6. Relation between $\Delta \delta$ and $\operatorname{Pr}(\text { fod })_{3} / \mathrm{MMA}$ unit. Numbers in the figure are assigned as following sequences.

$\alpha-\mathrm{CH}_{3}$ of the MMA unit: $1, \mathrm{M}-\mathrm{M}-\mathrm{M} ; 2, \mathrm{M}-$ $\mathrm{M}-\mathrm{I}+\mathrm{I}-\mathrm{M}-\mathrm{M} ; 3, \mathrm{I}-\mathrm{M}-\mathrm{I}$.

$\mathrm{CH}_{3}$ of the IP unit: $4, \mathrm{M}-\mathrm{I}-\mathrm{M} ; 5, \mathrm{M}-\mathrm{I}-\mathrm{I}+$ I-I-M; 7, I-I-I.

$\mathrm{HC}=$ of the IP unit: $6, \mathrm{M}-\mathrm{I}-\mathrm{M} ; 8, \mathrm{M}-\mathrm{I}-\mathrm{I}+$ I-I-M; 9, I-I-I.

and the olefin protons of the IP units were separated into three major peaks. These separated peaks are considered to be due to differences in the monomer sequence distributions.

In Figure 6 the differences $(\Delta \delta)$ between the lanthanide induced shifts and the original chemical shifts were plotted against the amount of $\operatorname{Pr}(\mathrm{fod})_{3}$ added, giving a linear relation be- tween the differences and the reagent added. In general, the lanthanide NMR shift reagent is complexed with the carbonyl group of the MMA unit. Therefore, the induced shift will be greater when the proton is close to the carbonyl group and/or is surrounded by MMA units. Previous studies on lanthanide shift reagent added to poly(methyl methacrylate) indicated $^{10,11}$ that the difference in stereochemical configuration on a sequence distribution of poly(methyl methacrylate) induced by a shift reagent was very small. Therefore, it is likely that the separation of peaks by the addition of $\operatorname{Pr}(\mathrm{fod})_{3}$ is caused by monomer sequence distributions. The sequence distributions thus obtained are shown in Figure 6, where $M$ and $I$ mean MMA and IP, respectively. These distributions were compared with those calculated according to a first-order Markov chain model with the assumption that the monomer reactivity ratios are $r_{\mathrm{IP}}=0.625$ and $r_{\mathrm{MMA}}=0.26$. As summarized in Table III, calculated and observed sequence distributions are in good agreement. The difference in monomer reactivity ratio between MMA-IP and MMA-IP$d_{4}$ is within the range of experimental error. This means that the deuterium isotope effect is negligible.

Acknowledgement. The authors are grateful to Mr. N. Nakayama for the measurement of NMR spectrum. 


\section{REFERENCES}

1. J. Furukawa, E. Kobayashi, and Y. Iseda, Bull. Inst. Chem. Res., Kyoto Univ., 47, 222 (1968); Polymer J., 1, 155 (1970).

2. J. Furukawa, E. Kobayashi, Y. Iseda, and Y. Arai, ibid., 1, 442 (1970).

3. T. Suzuki, Y. Takegami, J. Furukawa, E. Kobayashi, and Y. Arai, ibid., 4, 657 (1973).

4. J. Furukawa, E. Kobayashi, K. Uratani, Y. Iseda, J. Umemura, and T. Takenaka, ibid., 4, 358 (1973).

5. E. Oikawa and K. Yamamoto, ibid., 1, 669 (1970); E. Oikawa, A. Toki, and T. Suzuki, Kobunshi Kagaku (Chem. High Polymers), 30, 22 (1973).

6. B. Patnaik, A. Takahashi, N. G. Gaylord, $J$. Macromol. Sci. Chem., A4, 143 (1970).

7. J. C. Bevington and J. R. Ebdor, Makromol. Chem., 153, 173 (1972).

8. G. A. Lindsay, E. R. Santee, and H. J. Har- wood, Polym. Preprints 1973, 000.

9. C. C. Hinckley, J. Amer. Chem. Soc., 91, 5160 (1969).

10. J. E. Guillet, I. R. Peat, and W. F. Reynolds, Tetrahedron Lett., 1971, 3493.

11. S. Amiya, I. Ando, and R. Chûjô, Polymer J., 4, 385 (1973).

12. A. Yamamoto, K. Morifuji, S. Ikeda, T. Saito, Y. Uchida, and A. Misono, J. Amer. Chem. Soc., 90, 1878 (1968).

13. J. Furukawa, E. Kobayashi, and Y. Arai, Polymer J., 6, 489 (1974).

14. H. L. Frisch, C. L. Mallows, F. Heatley, and F. A. Bovey, Macromolecules, 1, 533 (1968).

15. K. Itoh and Y. Yamashita, J. Polym. Sci., Part $A-1,3,2165$ (1965).

16. J. Furukawa, E. Kobayashi, S. Nagata, and T. Moritani, The 22nd Annual Meeting of High Polymer, Kyoto, May, 1973; J. Polym. Sci., Part $A-1$, in press. 